\title{
Multidisciplinary Studies on Rotavirus-Human Milk Oligosaccharide Interactions
}

\author{
Sasirekha Ramani
}

$\mathbf{R}$ OTAVIRUS IS A LEADING cause of severe dehydrating gastroenteritis in children younger than 5 years. Despite the introduction of live, attenuated oral vaccines in 2006 , there are still $>200,000$ rotavirus-associated deaths in children worldwide, with the majority occurring in subSaharan African and southeast Asian countries. ${ }^{1}$ In neonates ( $<4$ weeks of age), rotavirus infections are predominantly asymptomatic, although association with severe gastrointestinal presentations such as feed intolerance and necrotizing enterocolitis have been described. ${ }^{2-4}$ Neonatal rotavirus infections are often caused by unusual strains that are geographically restricted. ${ }^{5}$ Studies in southern India show that the predominant rotavirus strain infecting neonates is G10P[11]. ${ }^{3}$

Rotaviruses are triple-layer particles; the outer capsid consists of the glycoprotein VP7 and the protease-sensitive spike protein VP4. ${ }^{6,7}$ During infection, the VP4 spike is cleaved into two fragments, VP5* and VP8*. The VP8* domain interacts with cellular glycans and mediates initial attachment to host cells. Sialic acid has traditionally been considered the key mediator of interactions for VP8*; however, recent data indicate that the VP8* of many human rotavirus strains can bind nonsialylated glycoconjugates called histo-blood group antigens (HBGAs). ${ }^{8-14}$

Human neonatal G10P[11] viruses are naturally occurring bovine-human reassortant strains and have a bovine VP8*spike. Potential binding partners for the P[11] VP8* were identified using a glycan array screen comprising $>600$ cellular glycans. ${ }^{14} \mathrm{P}[11]$ VP8* was found to specifically bind glycans with the Gal $\beta 1-4 \mathrm{GlcNAc}$ (LacNAc) motif, a precursor for type II HBGA (Fig. 1A). ${ }^{14}$ Expression of H-type HBGA in Chinese hamster ovary cells significantly enhanced G10P[11] infectivity (Fig. 1B), providing biological relevance to the glycan array results. The binding of P[11] VP8* to these
A

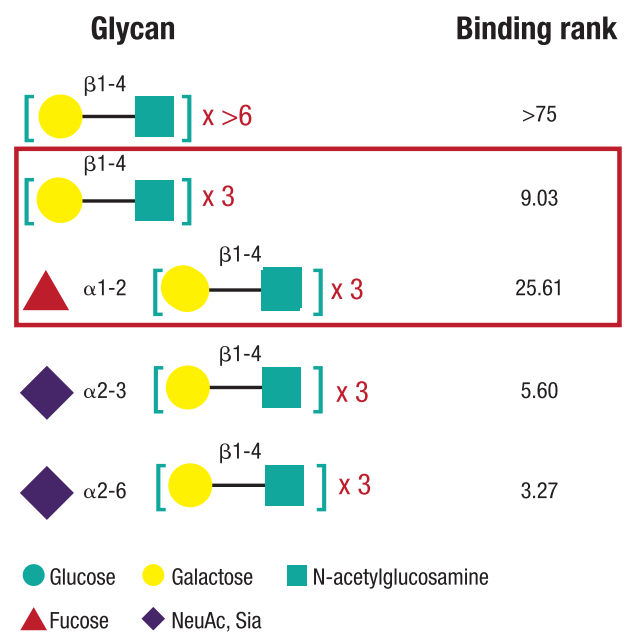

B
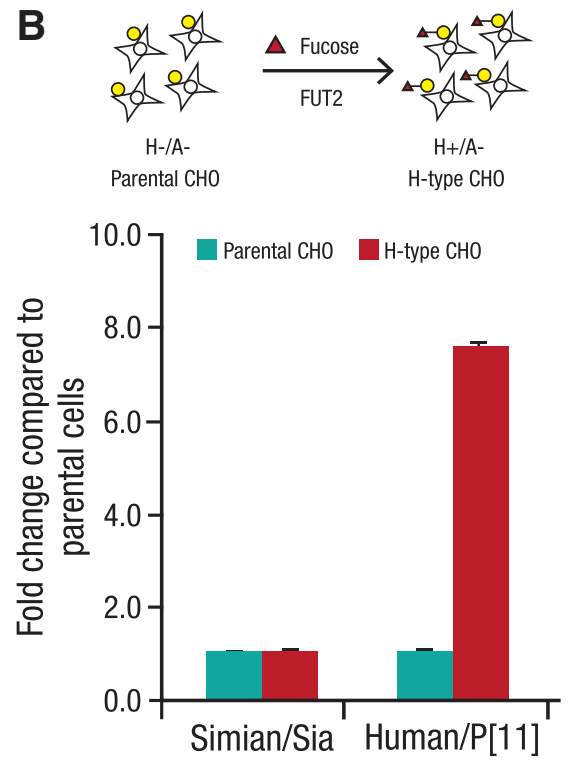

FUT, fucosyltransferase.

FIG. 1. (A) The G10P[11] VP8* domain binds to glycans with the LacNAc motif. ${ }^{14}$ (B) Expression of H-type HBGA enhanced human neonatal G10P[11] infectivity in Chinese Hamster Ovary (CHO) cells.

Assistant Professor, Department of Molecular Virology and Microbiology, Baylor College of Medicine, Houston, Texas. 
Table 1. Differences in Milk Glycan Binding

\begin{tabular}{lll}
\hline Species & \multicolumn{1}{c}{$\begin{array}{c}\text { P[11] VP8* } \\
\text { binding }\end{array}$} & $\begin{array}{c}\text { Predominant glycans } \\
\text { in milk }\end{array}$ \\
\hline Bovine & Type II & Type II \\
Human & Type I and type II & Type I, some type II \\
\hline Differences in milk glycan binding. ${ }^{11,19}$ &
\end{tabular}

Differences in milk glycan binding. ${ }^{11}$

precursor glycans that are developmentally regulated may explain the predilection of this strain for neonates.

Similar glycan structures are present in human milk as human milk oligosaccharides (HMOs). Binding of P[11]VP8* to HMOs was tested using a shotgun milk glycan array developed using pooled donor milk samples. ${ }^{15}$ Although the VP8* spike protein of the human neonatal G10P[11] virus is of bovine origin, VP8* from human and bovine G10P[11] strains bind different glycans in human milk. The human G10P[11] VP8* binds both type I and type II HMOs, whereas the bovine P[11] VP8* binds only to type II HMOs (Table 1). ${ }^{15}$ Crystallographic studies demonstrate that this difference in binding may be mediated by subtle variations in the glycan binding domain that prevent interaction with type I glycans in the bovine P[11] VP8*. ${ }^{9}$ Since bovine milk contains predominantly type II glycans and human milk contains type 1 and type II glycans, it is possible that the bovine G10P[11] strain may have evolved to recognize both types of glycans and infect human neonatal hosts.

Current studies using the G10P[11] strain are testing the hypothesis that complex interactions between intestinal glycans, and HMOs, affect neonatal susceptibility to rotavirus infections. Specific HMOs have been shown to reduce infectivity of two clinically important rotavirus strains that affect older infants. ${ }^{16}$ The development of nontransformed human intestinal epithelial cultures, called human intestinal enteroids, provides a new physiologically relevant tool to study the molecular basis of rotavirus-HMO interactions. ${ }^{17}$

\section{References}

1. Tate JE, Burton AH, Boschi-Pinto C, et al. Global, regional, and national estimates of rotavirus mortality in children <5 years of age, 2000-2013. Clin Infect Dis 2016; 62(suppl 2):S96-S105.

2. Dearlove J, Latham P, Dearlove B, Pearl K, Thomson A, Lewis IG. Clinical range of neonatal rotavirus gastroenteritis. Br Med J (Clin Res Ed) 1983;286:1473-1475.

3. Ramani S, Sowmyanarayanan TV, Gladstone BP, Bhowmick K, Asirvatham JR, Jana AK, Kuruvilla KA, Kumar M, Gibikote S, Kang G. Rotavirus infection in the neonatal nurseries of a tertiary care hospital in India. Pediatr Infect Dis J 2008;27:719-723.
4. Sharma R, Hudak ML, Premachandra BR, Stevens G, Monteiro CB, Bradshaw JA, Kaunitz AM, Hollister RA. Clinical manifestations of rotavirus infection in the neonatal intensive care unit. Pediatr Infect Dis J 2002;21: 1099-1105.

5. Haffejee IE. Neonatal rotavirus infections. Rev Infect Dis 1991;13:957-962.

6. Dormitzer PR, Sun ZY, Wagner G, et al. The rhesus rotavirus VP4 sialic acid binding domain has a galectin fold with a novel carbohydrate binding site. EMBO J 2002;21:885897.

7. Settembre EC, Chen JZ, Dormitzer PR, et al. Atomic model of an infectious rotavirus particle. EMBO J 2011; 30:408-416.

8. Hu L, Crawford SE, Czako R, et al. Cell attachment protein VP8* of a human rotavirus specifically interacts with Atype histo-blood group antigen. Nature 2012;485:256-259.

9. Hu L, Ramani S, Czako R, et al. Structural basis of glycan specificity in neonate-specific bovine-human reassortant rotavirus. Nat Commun 2015;6:8346.

10. Bohm R, Fleming FE, Maggioni A, et al. Revisiting the role of histo-blood group antigens in rotavirus host-cell invasion. Nat Commun 2015;6:5907.

11. Huang P, Xia M, Tan M, et al. Spike protein VP8* of human rotavirus recognizes histo-blood group antigens in a type-specific manner. J Virol 2012;86:4833-4843.

12. Liu Y, Huang P, Jiang B, et al. Poly-LacNAc as an agespecific ligand for rotavirus $\mathrm{P}[11]$ in neonates and infants. PLoS One 2013;8:e78113.

13. Liu Y, Huang P, Tan M, et al. Rotavirus VP8*: phylogeny, host range, and interaction with histo-blood group antigens. J Virol 2012;86:9899-9910.

14. Ramani S, Cortes-Penfield $\mathrm{NW}, \mathrm{Hu} \mathrm{L}$, et al. The VP8*domain of neonatal rotavirus strain G10P[11] binds to type II precursor glycans. J Virol 2013;87:7255-7264.

15. Yu Y, Lasanajak Y, Song X, et al. Human milk contains novel glycans that are potential decoy receptors for neonatal rotaviruses. Mol Cell Proteomics 2014;13:2944-2960.

16. Laucirica DR, Triantis V, Schoemaker R, et al. Milk oligosaccharides inhibit human rotavirus infectivity in MA104 cells. J Nutr 2017;147:1709-1714.

17. Saxena K, Blutt SE, Ettayebi K, et al. Human intestinal enteroids: A new model to study human rotavirus infection, host restriction, and pathophysiology. J Virol 2015; 90:43-56.

Address correspondence to: Sasirekha Ramani, PhD

Department of Molecular Virology and Microbiology Baylor College of Medicine 1 Baylor Plaza Houston, TX 77030

E-mail: ramani@bcm.edu 